\title{
Article \\ Identification of an Oxidosqualene Cyclase Gene Involved in Steroidal Triterpenoid Biosynthesis in Cordyceps farinosa
}

\author{
Gi-Hong An ${ }^{1,+}{ }^{\mathbb{D}}$, Jae-Gu Han ${ }^{1,+}$, Hye-Sung Park ${ }^{1}$, Gi-Ho Sung ${ }^{2}$ and Ok-Tae Kim ${ }^{1, *(\mathbb{D})}$ \\ 1 Department of Herbal Crop Research, National Institute of Horticultural and Herbal Science, \\ Rural Development Administration, Eumseong 27709, Korea; agiho@korea.kr (G.-H.A.); \\ jaeguhan@gmail.com (J.-G.H.); hyesung2@korea.kr (H.-S.P.) \\ 2 Catholic Kwandong University International St. Mary's Hospital, Incheon 22711, Korea; \\ sung97330@gmail.com \\ * Correspondence: kimot99@hanmail.net; Tel.: +82-43-871-5730 \\ + These authors contributed equally to this study.
}

check for updates

Citation: An, G.-H.; Han, J.-G.; Park, H.-S.; Sung, G.-H.; Kim, O.-T Identification of an Oxidosqualene Cyclase Gene Involved in Steroidal Triterpenoid Biosynthesis in Cordyceps farinosa. Genes 2021, 12, 848. https:// doi.org/10.3390/genes12060848

Academic Editor: Quan Zou

Received: 23 April 2021

Accepted: 28 May 2021

Published: 31 May 2021

Publisher's Note: MDPI stays neutral with regard to jurisdictional claims in published maps and institutional affiliations.

Copyright: (c) 2021 by the authors. Licensee MDPI, Basel, Switzerland. This article is an open access article distributed under the terms and conditions of the Creative Commons Attribution (CC BY) license (https:// creativecommons.org/licenses/by/ $4.0 /)$.
Abstract: Various fungi including Cordyceps farinosa, an entomopathogenic fungus, can produce steroidal triterpenoids. Protostadienol (protosta-17(20)Z,24-dien-3 $\beta$-ol) is a precursor of steroidal triterpenoid compounds. To identify oxidosqualene cyclase (OSC) gene candidates involved in triterpenoid biosynthesis, genome mining was performed using Illumina sequencing platform. In the sequence database, two OSC genes, $C f a O S C 1$ and $C f a O S C 2$, in the genome of $C$. farinosa were identified. Predicted amino-acid sequences of CfaOSC2 shared $66 \%$ similarities with protostadienol synthase (OSPC) of Aspergillus fumigatus. Phylogenetic analysis showed a clear grouping of CfaOSC2 in the OSPC clade. Function of CfaOSC2 was examined using a yeast INVSc1 heterologous expression system to endogenously synthesize 2,3-oxidosqualene. GC-MS analysis indicated that CfaOSC2 produced protosta-13(17),24-dien-3 $\beta$-ol and protostadienol at a 5:95 ratio. Our results demonstrate that CfaOSC2 is a multifunctional triterpene synthase yielding a predominant protostadienol together with a minor triterpenoid. These results will facilitate a greater understanding of biosynthetic mechanisms underlying steroidal triterpenoid biosynthesis in C. farinosa and other fungi.

Keywords: Cordyceps farinosa; fusidic acid; oxidosqualene cyclase; protostadienol synthase; triterpenoid

\section{Introduction}

Steroidal triterpenoids such as ganoderic acid, pachymic acid, fusidic acid, and helvolic acid are produced by fungal cells. These compounds can protect fungal cells against other pathogens. Ganoderic acid and pachymic acid have anti-cancer, immuneenhancing, and anti-inflammation effects [1-3]. Fusidic acid and helvolic acid are widely used as antibiotics against Gram-positive bacteria. The biosynthesis mechanism of fusidic acid and helvolic acid has been recently elucidated using a heterologous expression system [4,5]. However, the pathway of other steroidal triterpenoid biosynthesis is not clear yet.

A number of studies have suggested that lanosterol and protostadienol (protosta17(20)Z,24-dien-3 $\beta$-ol) are precursors of steroidal triterpenoid compounds [6,7]. In the case of triterpene biosynthesis in fungal cells, lanosterol synthase (OSLC) and protostadienol synthase (OSPC) belonging to a class of oxisqualene cyclases (OSCs) are involved in the biosynthesis of lanostane-type and fusidane-type triterpenoid, respectively (Figure 1). It has been reported that these OSCs are key enzymes that regulate biosynthesis [8]. In both fungi and animals, OSLC is responsible for producing sterol precursors for cholesterol and ergosterol. OSLC genes from several fungal species, animals, plants, and microorganisms have been characterized [9-13]. Interestingly, genomes of some fungi encode more than one OSC. Other OSCs can also produce triterpenoid secondary metabolites via their OSC 
function. So far, only one OSPC gene has been characterized in A. fumigatus through a heterologous expression system in yeast cells [6]. OSPC gene from other organisms has not been characterized yet.

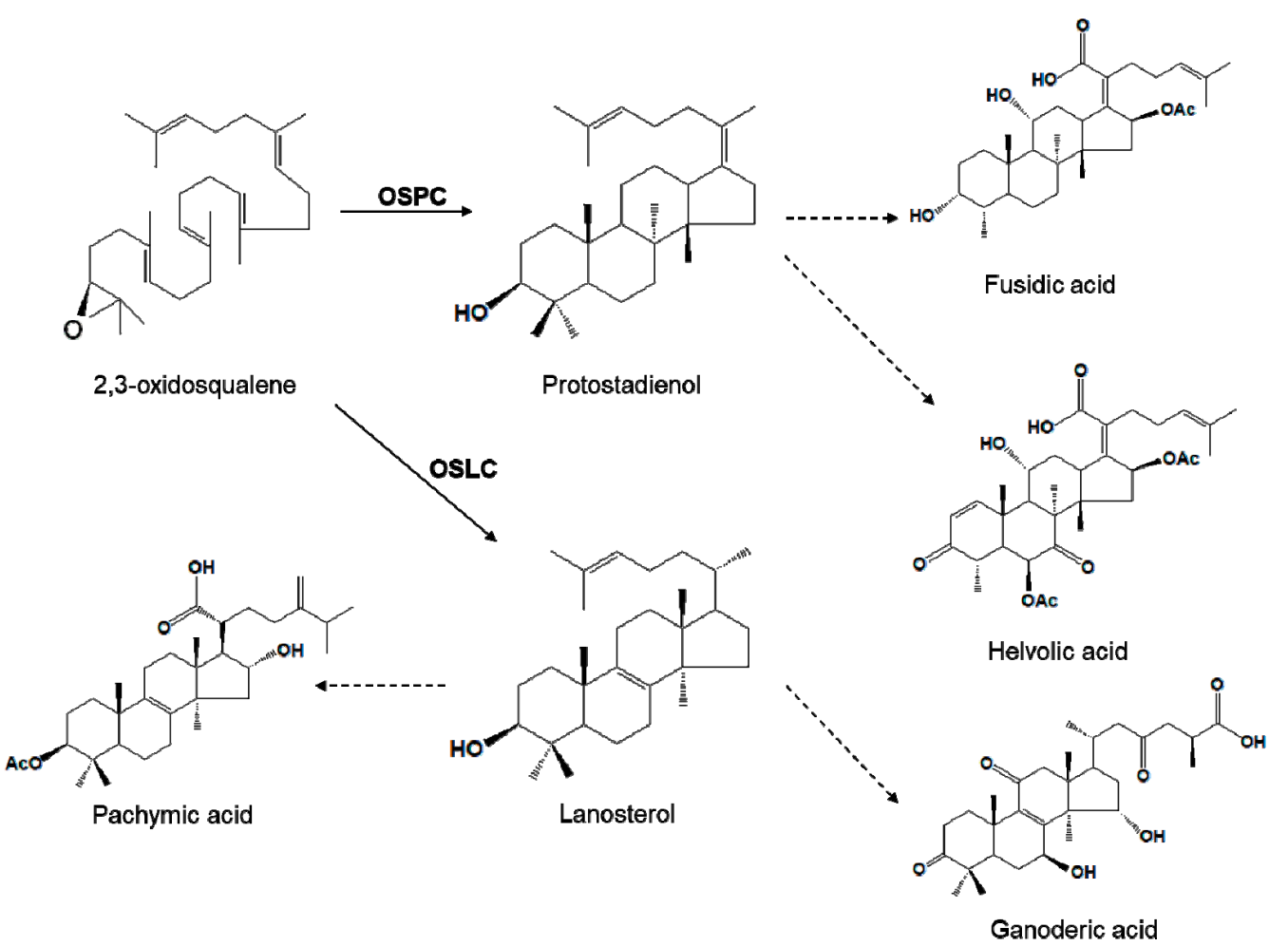

Figure 1. Putative biosynthesis pathway of steroidal triterpenoids in fungi. Dot arrows mean multiple steps.

The species Cordyceps farinosa belongs to the Cordycipitaceae family in the Hypocreales order of Ascomycota and is well-known as entomopathogenic fungi with a worldwide distribution in temporate and tropical zones [14]. These entomopathogenic fungi can also produce a variety of chemicals that cause severe adverse reactions in other organisms, including bacteria and other animals. Sarocladium oryzae, Acremonium fusidioides, Metarhizium anisopliae, and Aspergillus fumigatus are fungi that can produce fusidanes as steroidal antibiotics [15]. In particular, Cordyceps kogane can produce fusidic acid that is structurally similar to fusidane [16].

To discover genes related to triterpenoid biosynthesis, Cordyceps farinosa mycelium was chosen as a biosynthesis model of steroidal triterpenoid. We chose $C$. farinosa because, in the preliminary comparative genome analysis of Hypocrealean species, we recognized that a gene cluster of $C$. farinosa was similar to a well-known gene cluster related to helvolic acid biosynthesis in $A$. fumigatus. In the present study, whole genome sequences of $C$. farinosa KMCC47486 were analyzed and two OSC genes, lanosterol synthase and protostadienol synthase, were found. We isolated a gene encoding a putative protostadienol synthase from C. farinosa mycelium. Its function was then determined using a heterologous expression system in yeast along with product analysis using GC-MS. In addition, a gene cluster including OSPC gene was identified. It consisted of seven genes highly conserved in A. fumigauts for helvolic acid biosynthesis. We also discussed triterpenoids produced by discovering genes that matched with the gene cluster related to helvolic acid biosynthesis.

\section{Materials and Methods}

\subsection{Strain of C. farinosa}

A strain of C. farinosa (KACC47486) was obtained from Korean Agricultural Culture Collection (KACC), Rural Development Administration (RDA), Republic of Korea. The 
mycelium of $C$. farinosa was cultured on PDA (Potato Dextrose Agar) medium at $25^{\circ} \mathrm{C}$ in darkness for 2 weeks. To identify the strain through sequence comparison of its ITS rDNA region, phylogenetic tree analysis was performed using the Neighbor-Joining method.

\subsection{Genome Sequencing and Assembly}

Genomic DNA was extracted from freeze-dried mycelium using a DNeasy Plant Mini Kit (Qiagen, Valencia, CA, USA) according to the manufacturer's instructions. Concentration and purity of the extracted genomic DNA were assessed using a NanoDrop ND-1000 spectrophotometer (Thermo-Fisher Scientific, Waltham, MA, USA). Next generation sequencing (NGS) library construction and sequencing were carried out by Macrogen Inc. (Seoul, Korea). Paired-end and mate-pair libraries with insert sizes of $300 \mathrm{bp}$ and $5 \mathrm{kbp}$, respectively, were prepared. Subsequently, NGS was carried out using an Illumina HiSeq platform (KAPA Biosystems, Roche, San Francisco, CA, USA) with a coverage of approximately $350 \times$. Generated sequence reads with low-quality scores or short lengths were filtered out using a FASTX Toolkit 0.0.13 (http://hannonlab.cshl.edu/fastx_toolkit (accessed on 28 May 2021)). Trimmed paired-end and mate-pair reads were then assembled using a Velvet 1.2.01 assembler [17]. All reads were deposited at NCBI, and can be accessed in the BioProject data base under project accession number PRJNA314175.

\subsection{Phylogenetic Analysis}

Amino acid sequences of OSCs in C. farinosa and its related species were extracted using tblastn of NCBI BLAST 2.2.28+ package [18] with a query sequence of A. fumigatus OSLC (XP_747936) against selected fungal genomes belonging to the order Hypocreales: Beauveria bassiana ARSEF2860 (PRJNA38719), Claviceps purpurea 20.1 (PRJEA76493), Cordyceps militaris CM01 (PRJNA41129), Epichloë festucae FI1 (PRJNA51625), Epichloë typhina E8 (PRJNA174036), Fusarium graminearum PH-1 (PRJNA243), Fusarium oxysporum Fo5176 (PRJNA68027), Fusarium verticillioides 7600 (PRJNA15553), Metarhizium acridum CQMa 102 (PRJNA38715), Metarhizium anisopliae ARSEF23 (PRJNA38717), Tolypocladium inflatum NRRL8044 (PRJNA73163), Trichoderma atroviride IMI206040 (PRJNA19867), Trichoderma reesei QM6a (PRJNA15571), Trichoderma virens Gv29-8 (PRJNA19983), Beauveria pseudobassiana KACC47484 (PRJNA314175), Beauveria sungii KACC47481 (PRJNA314175), Cordyceps pruinosa KACC44470 (PRJNA314175), Cordyceps farinosa KACC47486 (PRJNA314175), and Cordyceps tenuipes KACC47485 (PRJNA314175). Predicted amino acid sequences were aligned using Clustal Omega [19] with default settings and then manually adjusted. Phylogenies were inferred using neighbor joining (NJ) and maximum likelihood (ML) methods of a MEGA6 software [20]. Relative robustness of individual branches was estimated with 500 replicates using bootstrapping (BS).

\subsection{Generation of Plasmid Vectors}

Sequences for two C. farinosa genes were deposited in GenBank with accession numbers of MF972281 for CfaOSC1 and MF972287 for CfaOSC2. To amplify the CfaOSC2 gene, forward (5'-TGATGCCTGTCGCCGATATTGAC- $\left.3^{\prime}\right)$ and reverse (5'-TTATCCTTTGTA AAAGGACGCTC- $3^{\prime}$ ) primers were designed. PCR was then performed with an Ex-taq DNA polymerase (Takara, Tokyo, Japan) using the following cycling conditions: 30 cycles at $95^{\circ} \mathrm{C}$ for $1 \mathrm{~min}, 58^{\circ} \mathrm{C}$ for $1 \mathrm{~min}$, and $72^{\circ} \mathrm{C}$ for $2 \mathrm{~min}$; followed by a final extension step at $72{ }^{\circ} \mathrm{C}$ for $10 \mathrm{~min}$. The obtained fragment was purified and cloned into a pYES2.1 vector using a TOPO TA-expression kit (Invitrogen, Carlsbad, CA, USA), enabling the construction of an expression plasmid pYES-CfaOSC2 in yeast cells. After sequence (pYES-CfaOSC2) confirmation, the plasmid was used for yeast transformation.

\subsection{Functional Expression of CfaOSC2 in Yeast}

Functional characterization was carried out in yeast strain INVSc1 purchased from Invitrogen (Carlsbad, CA, USA). Yeast transformation and insert-DNA overexpression were carried out as described by Kushiro et al. [21]. Single clones including pYES-CfaOSC2 were 
incubated in $15 \mathrm{~mL}$ of a synthetic complete medium containing $2 \%$ glucose without uracil at $30{ }^{\circ} \mathrm{C}$ with shaking at $220 \mathrm{rpm}$ for two days. After three-day induction with $2 \%$ galactose, cells from two $50 \mathrm{~mL}$ conical tubes were collected into one tube, refluxed with $8 \mathrm{~mL}$ of $20 \%$ potassium hydroxide $(\mathrm{KOH})$ and $50 \%$ ethanol $(\mathrm{EtOH})$, and extracted three times with hexane at the same volume. Extracts were concentrated under a stream of nitrogen gas $\left(\mathrm{N}_{2}\right)$ and resuspended in $1 \mathrm{~mL}$ of chloroform $\left(\mathrm{CHCl}_{3}\right)$. Subsequently, $100 \mu \mathrm{L}$ of the extract was transferred into a new vial and concentrated under a stream of $\mathrm{N}_{2}$ at $70^{\circ} \mathrm{C}$. For GC-MS analysis, the extract was silylated with $50 \mu \mathrm{L}$ of $\mathrm{N}, \mathrm{O}$-bis(trimethylsilyl)trifluoroacetamide (BSTFA) and $50 \mu \mathrm{L}$ of pyridine for $30 \mathrm{~min}$ at $70{ }^{\circ} \mathrm{C}$.

\subsection{GC-MS Analysis}

GC-MS analyses were run under the same conditions as described by Wang et al. [22]. Briefly, a $1-\mu \mathrm{L}$ aliquot of the solution was analyzed using a $7890 \mathrm{~N}$ gas chromatography (Agilent Technologies, Santa Clara, CA, USA) equipped with a 5973-inert mass spectrometer (MS) detector (Agilent Technologies, Santa Clara, CA, USA) and an Agilent HP-5 capillary column (length of $30 \mathrm{~m}$, i.d. of $250 \mu \mathrm{m}$, film thickness of $0.25 \mu \mathrm{m}$ ). The injection temperature was set at $50{ }^{\circ} \mathrm{C}$ for $2 \mathrm{~min}$. The column-temperature program was as follows: $40{ }^{\circ} \mathrm{C} / \mathrm{min}$ ramp to $200{ }^{\circ} \mathrm{C}$, a hold at $200{ }^{\circ} \mathrm{C}$ for $2 \mathrm{~min}$, followed by an increase to $320^{\circ} \mathrm{C}$ at a rate of $3{ }^{\circ} \mathrm{C} / \mathrm{min}$; and lastly a hold at $320^{\circ} \mathrm{C}$ for $30 \mathrm{~min}$. Triterpenoids were identified by comparison with authentic extracts from yeast cells co-expressing AfuOSC3 (A. fumigatus protostadienol synthase) and CYP5081A1 (A. fumigatus cytochrome P450 oxidase) as reported by Mitsuguchi et al. [7]. The constructed vector of pESC(-Ura)AfuOSC3/CYP5081A1 was kindly provided by Dr. Tetsuo Kushiro (Meiji University, Tokyo, Japan). As previously described, the same protocol was applied for the transformation and culturing of yeast strain INVSc1 with the vector.

\subsection{Synteny Analysis}

The OSC gene cluster of A. fumigatus Af293 proposed by Lodeiro et al. [6] was retrieved from GenBank (XP_751348-XP_751356), including a series of genes catalyzing monooxygenation, dehydrogenation, and acyl transfer to convert protostadienol into helvolic acid. Based on the result of our phylogenetic analysis, the synteny of OSC gene cluster of $A$. fumigatus was searched within the three selected hypocrealean species., viz. M. anisopliae, B. sungii, and C. farinosa (Group III in Figure 4). Orthologous regions were identified using the reciprocal best hit $(\mathrm{RBH})$ approach [23]. First, local databases were created from genome sequences of C. farinosa. After BLAST-searching AfuOSC3 gene clusters in databases, nucleotide sequences highly matched with protein sequences of AfuOSC3 were extracted from each genome. They were then conversely queried against the AfuOSC3 gene cluster. In each pair-wise comparison, reciprocally best-matched genes (or regions) were regarded as orthologs.

\section{Results and Discussion}

\subsection{Identification of C. farinosa}

The mycelium of putative C. farinosa, which was provdied by the KACC (Korean Agriculture Culture Collection), was cultured on PDA medium for 2 weeks (Figure 2a). To identify the strain, the fungal source was analyzed by ITS gene sequencing. The BLAST search of the ITS sequence of the strain showed the highest similarity to Cordyceps and the phylogenetic tree was constructed using MEGA 5.2 software. As shown in Figure 2b, this strain clearly was grouped with $C$. farinosa. In addition, Isaria farinosa as the scientific name has long been used for this fungus. However, the genetic position of Isaria was changed to the Cordyceps by the principal of priority because of the discontinuance of dual nomenclature for pleomorphic fungi in 2011 [24]. After confirmation of the strain, we performed genome sequencing. 


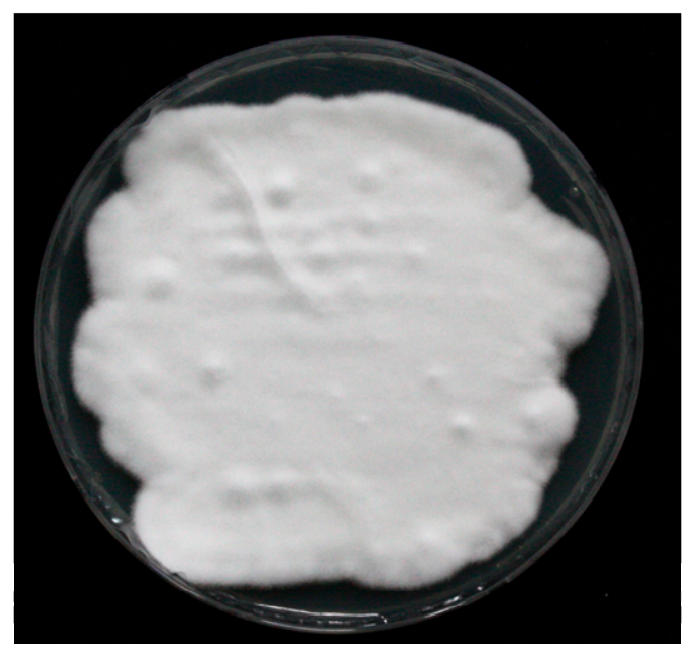

(a)

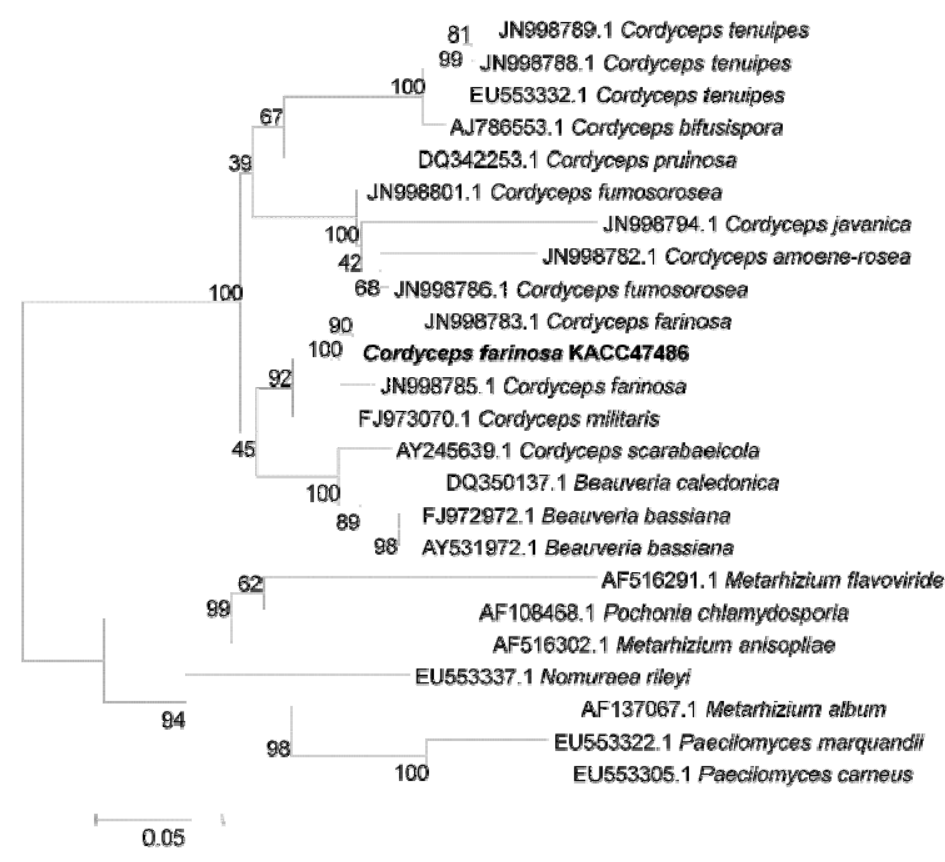

(b)

Figure 2. Identification of Cordyceps farinosa (KACC47486). (a) Strain of C. farinosa (KACC47486) cultured on PDA medium for two weeks; (b) Phylogenetic analysis using Neighbor-Joining method based on partial sequences of the ITS rDNA region.

\subsection{Sequence Features of Two OSC Genes}

Mass DNA-sequence data were produced using an Illumina sequencing platform to identify OSC genes involved in triterpenoid biosynthesis. In the sequence database, two OSC genes, CfaOSC1 (C. farinosa lanosterol synthase) and CfaOSC2 (C. farinosa protostadienol synthase), were identified from the genome of $C$. farinosa. Both genes contained full-length cDNAs. Open reading frames (ORFs) of $C f a O S C 1$ and $C f a O S C 2$ were 2223 bp and $2205 \mathrm{bp}$ in length, respectively. These ORFs were predicted to encode CfaOSC1 and CfaOSC2 proteins having 741 and 735 amino acids with masses of $84.407 \mathrm{kDa}$ and $82.615 \mathrm{kDa}$, respectively. Deduced amino acid sequences of CfaOSC1 (70\%) and CfaOSC2 $(66 \%)$ were orthologous to lanosterol synthase (Afu5g04080) and protostadienol synthase (AfuOSC3) of A. fumigatus, respectively. As shown in Figure 3, CfaOSC1 and CfaOSC2 contained repeats of the QW motif [25], a typical feature of the triterpene-synthase superfamily. CfaOSC2 consisted of ${ }^{699}$ ACPGGMR ${ }^{705}$ motif in the C-terminal region. This motif is known to play a role in protostadienol formation [26].

To determine the relationship of these two CfaOSCs with other fungal OSCs, phylogenetic analysis was performed as to determine its relationships with other fungi (Figure 4). As expected, $\mathrm{CfaOSC} 1$ and $\mathrm{CfaOSC} 2$ were clearly grouped in OSLC and OSPC clades, respectively. Therefore, $\mathrm{CfaOSC} 2$ is likely to have function in protostadienol production. 


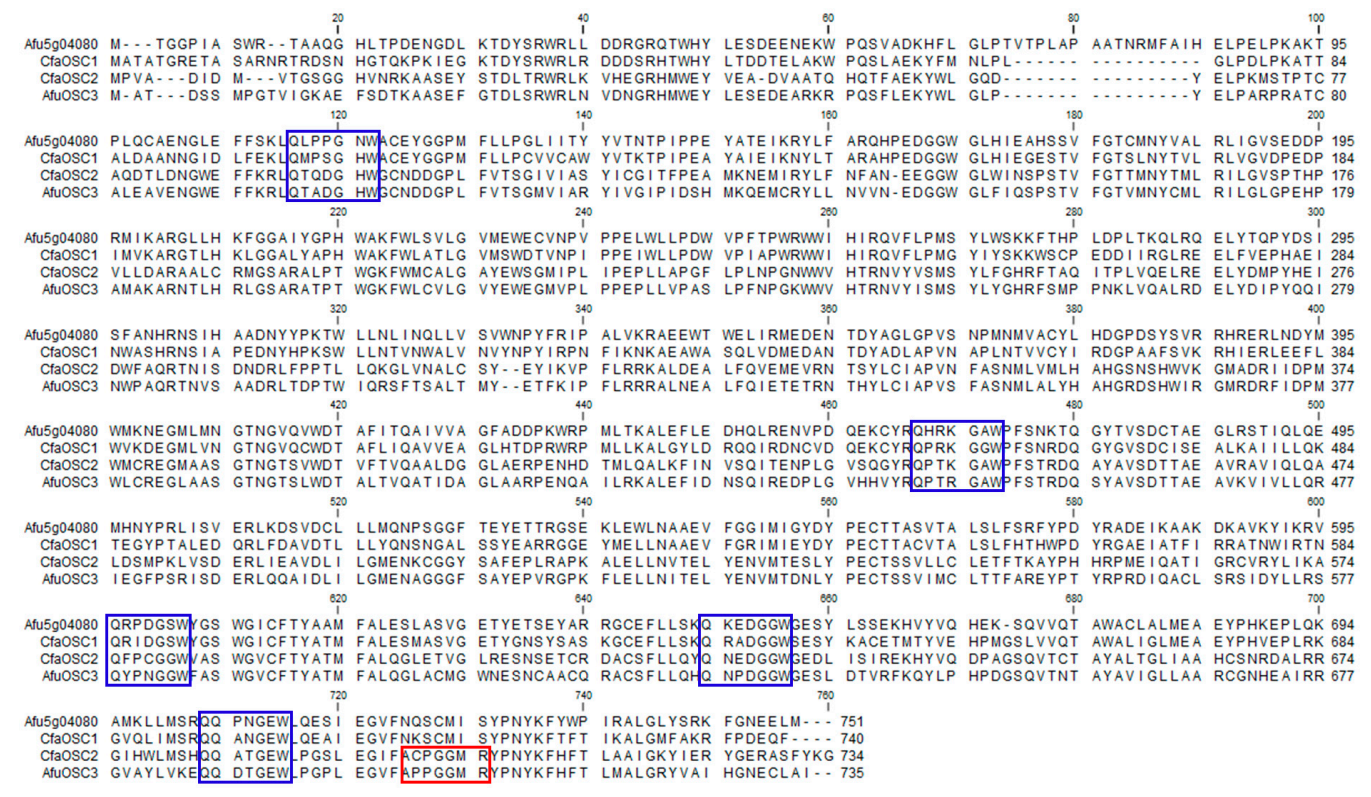

Figure 3. Alignment of amino-acid sequences of OSC genes in Cordyceps farinosa and Aspergillus fumigatus. Blue and red boxes indicate the QW(QXXXGXW) motif and the specific motif that plays a role in protostadienol production, respectively.
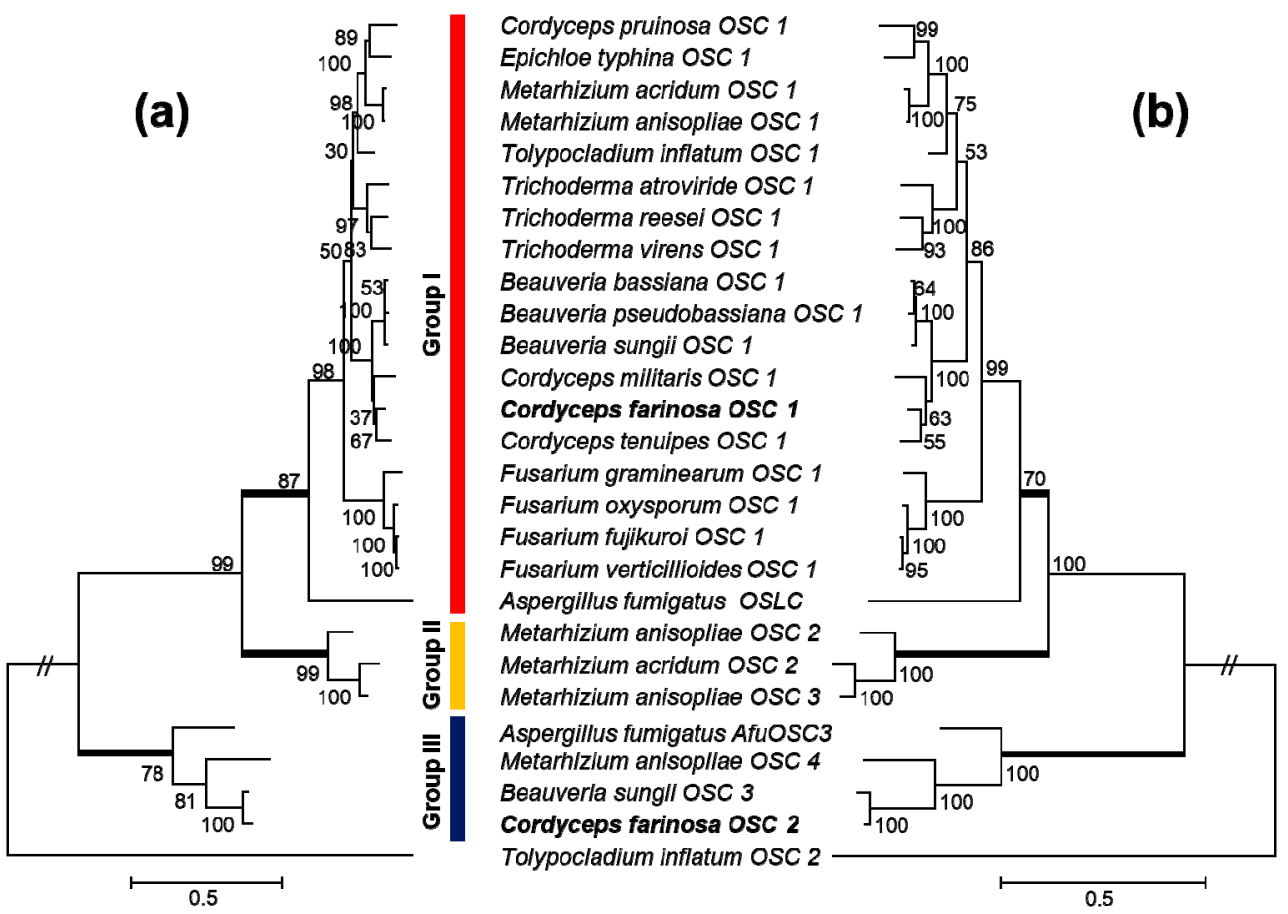

Figure 4. Phylogenetic tree of oxidosqualene cyclase (OSC) sequences extracted from selected Hypocreales species and inferred by neighbor joining (NJ, a) and maximum likelihood (ML, b) analyses. The number of changes among sequences is presented by branch length. The scale bar equals the number of nucleotide substitutions per site. Group I comprises genes homologous to lanosterol synthase (OSLC). Group III delimits orthologous of protostadienol synthase (OSPC). Three uncharacterized OSCs from Metarhizium spp. are nested in Group II.

\subsection{Functional Characterization of $\mathrm{CfaOSC} 2$ in Yeast}

Full-length cDNA of $C f a O S C 2$ was amplified using polymerase chain reaction (PCR) to elucidate the function of $C f a O S C 2$ gene in steroidal triterpenoid biosynthesis. The fulllength cDNA was cloned into a yeast expression vector pYES2.1 under the control of a 
GAL1 promotor. The $C f a O S C 2$ gene functionally was characterized using a heterologous expression system in yeast INVSc1 that could endogenously synthesize 2,3-oxidosqualene. After three days of galactose induction, yeast cells were harvested and then extracted with hexane. To check whether or not alcohol triterpenes were extracted from yeast cells, we added lupeol as a internal standard before the extraction. Results of gas chromatographymass spectrometry (GC-MS) analysis indicated that CfaOSC2-overexpressing yeast cells produced two products not present in control yeast cells carrying an empty vector pYES2 (Figure 5a). Peaks of yeast cells co-expressing AfuOSC3/CYP5081A1 as described by Mitsuguchi et al. [7] were used as authentic standards. Results of retention-time comparison indicated that these two products at a ratio of 5:95 were protosta-13(17),24-dien-3 $\beta$-ol (1) and protosta-17(20)Z,24-dien-3 $\beta$-ol (2, protostadienol), respectively. In addition, the pattern of mass fragments of these identified compounds in yeast cells overexpressing AfuOSC3/CYP5081A1 was compared. Results are shown in Figure 5b. Both peaks were alcohol triterpenes as they comprised the $m / z 498$ molecular ion which was trimethylsilylated (TMS). In addition, the characteristic fragment ions in MS spectrum of protostadienol were $m / z=191,339$ and 429, and these values were consistent with those reported by Ledro et al. [6] (Supplementary Figure S1). Therefore, CfaOSC2 is a multifunctional triterpene synthase producing compounds $\mathbf{1}$ and $\mathbf{2}$.

(a)

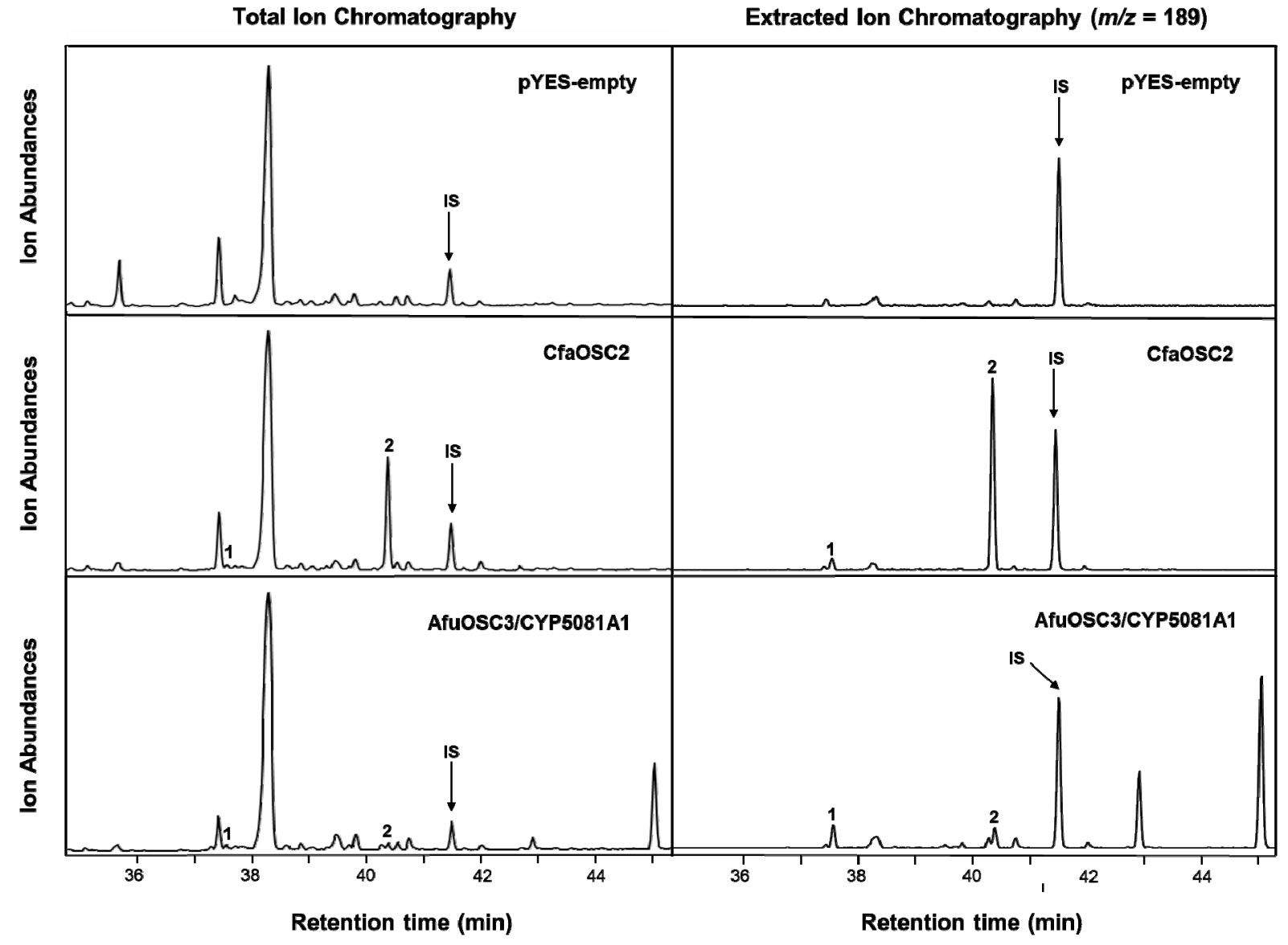

Figure 5. Cont. 


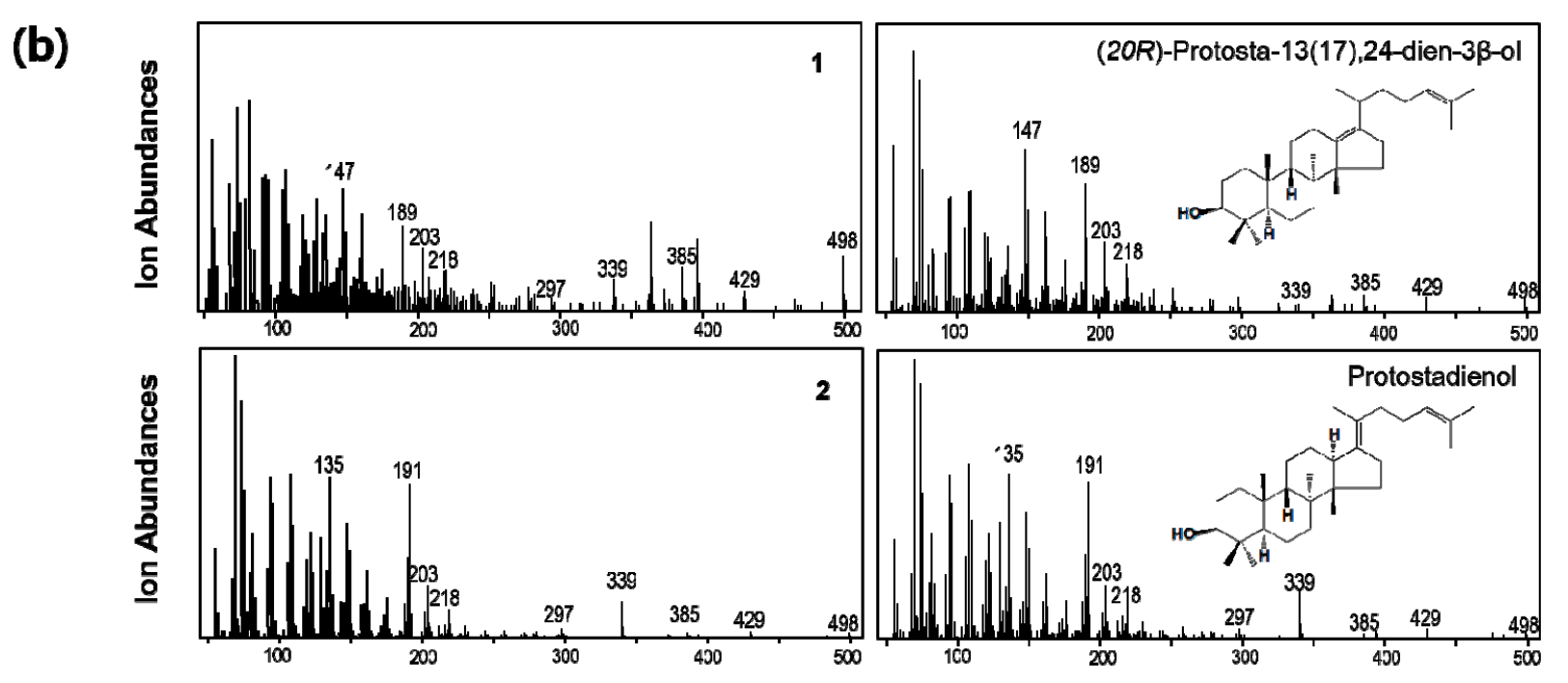

Figure 5. Identification of triterpenoids in CfaOSC2-overexpressing yeast cells by gas chromatography-mass spectrometry (GC-MS) analysis. (a) GC chromatograms from the strain expressing the CfaOSC2 gene, the control yeast strain harboring the pYES2 vector, and the authentic standards from the yeast strain co-expressing AfuOSC3/CYP5081A1 genes. IS means internal standard (Lupeol). (b) MS spectra of trimethylsilylated triterpenoids produced from the CfaOSC2-expressing strain (left) and the standard (right).

\subsection{Synteny Comparison of a Gene Cluster for Helvolic Acid Biosynthesis}

Whole fungi-genome sequences are available from public databases. Lodeiro et al. [6] and Mitsuguchi et al. [7] have identified a gene cluster related to helvolic acid biosynthesis that consists of OSPC, four cytochrome P450 (CYP) monooxygenases, two transferasefamily proteins, and two dehydrogenase genes. Nine of these gene-cluster genes have been recently characterized using a heterologous-expression system in Aspergillus oryzae NSAR1, resulting in the detection of helvolic acid together with 21 protostadienol derivatives [4]. We compared the gene cluster of A. fumigatus for helvolic acid biosynthesis with that of C. farinosa based on DNA sequences (Figure 6). A gene cluster of C. farinosa consists of seven genes, encoding one protostadienol synthase, three cytochrome P450 (CYP), one acyltransferase, one short-chain dehydrogenase/reductase and one ketosteroid dehydrogenase. Among seven genes, amino acids of five genes are highly similar to those of HelA (65\%), HelB1 (64\%), HelB2 (60\%), HelC (61\%) and HelB4 (66\%). However, amino acids of two genes encoding acyltransferase and ketosteroid dehydrogenase showed the low level of similarity to those of HelD2 (38\%) and HelE (26\%). The gene cluster of C. farinosa showed deletion of a CYP gene (Gene No. 5) and an acyltransferase gene (Gene No. 6) compared to the helvolic acid cluster. We performed HPLC analysis to determine helvolic acid in C. farinosa (Supplementary Figure S2). The result indicated that a peak which was not consistent with that of helvolic acid was detected. Therefore, the result might indicate that helvolic acid is not biosynthesized because of deletion of the two genes in C. farinosa. Based on these results, we depicted a putative pathway of triterpenoid biosynthesis in C. farinosa (Figure 7). We inferred that the final product of operating this gene cluster might be 16ß-acetyloxy-29-norprotosta-1,17(20)Z,24-trien-3-one-21-oic acid considering that those two genes (Gene No. 5 and Gene No. 6) were deleted in the gene cluster. Future studies are needed to identify all genes except OSPC gene using a heterologous expression system. 


\section{Scaffold_4}

A. fumigatus

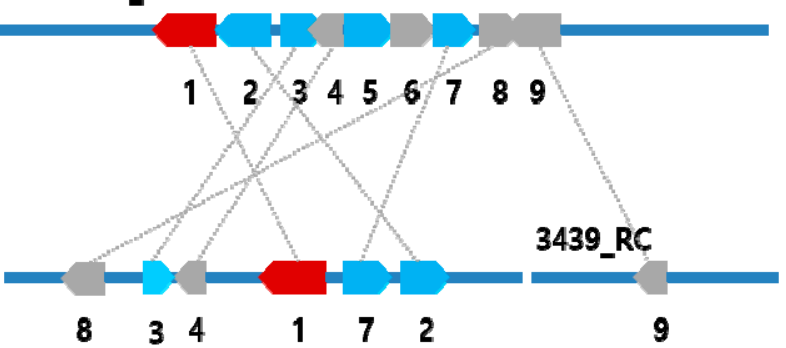

\section{No. Gene name Description (Accession No.)}

1 HelA

2 HelB1

3 HelB2

$4 \mathrm{HelC}$

5 HelB3

6 HelD1

7 HelB4

8 HelD2

Protostadienol synthase (XP_751356.1)

C. farinosa

Cytochrome P450 monooxygenase (XP_751355.1)

Cytochrome P450 monooxygenase (XP_751354.1)

Short chain dehydrogenase (XP_751353.1)

Cytochrome P450 monooxygenase (XP_751352.2)

Transferase family protein (XP_751351.1)

Cytochrome P450 monooxygenase (XP_751350.1)

9 HelE

Transferase family protein (XP_751349.1)

Extracellular 3-ketosteroid 1-dehydrogenase (XP_751348.1)

Figure 6. Synteny comparison of two gene clusters involved in triterpenoid biosynthesis.
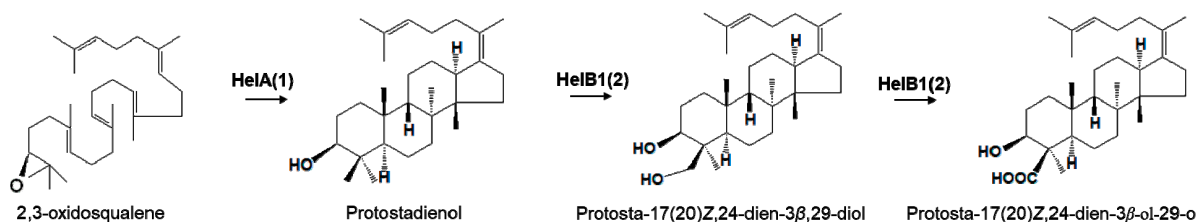

Protosta-17(20)Z,24-dien-3ß,29-diol

Protosta-17(20)Z,24-dien-3 $\beta$-ol-29-oic acid

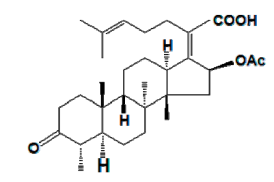

16ß-acetyloxy-29-norprotosta17(20)Z,24-dien-3-one-21-oic acid

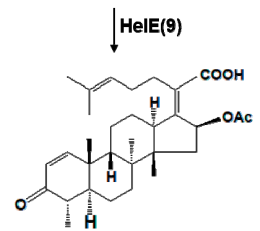

16ß-acetyloxy-29-norprotosta1,17(20)Z,24-trien-3-one-21-oic acid

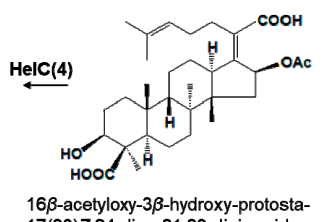

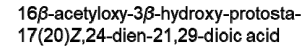

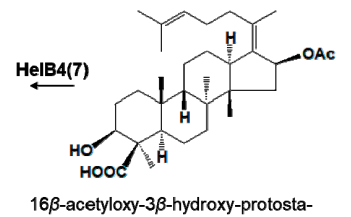
16 $\beta$-acetyloxy-3 $\beta$-hydroxy-protosta-
$17(20) E, 24-$ dien-29-oic acid
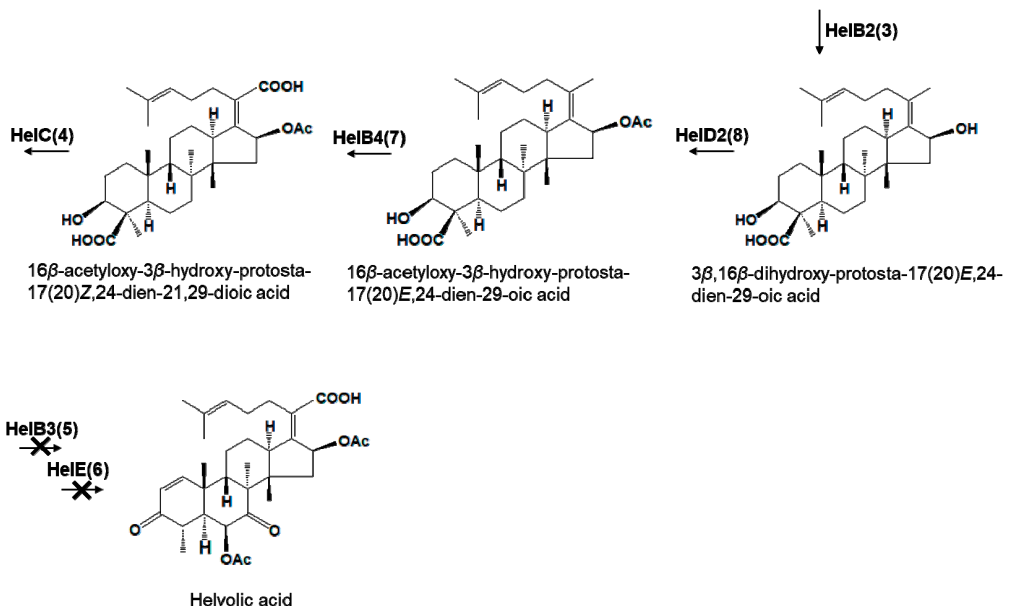

Figure 7. Putative pathways of triterpenoid biosynthesis in C. farinosa.

\section{Conclusions}

This study revealed that the $C f a O S C 2$ gene encoded an enzyme that could catalyze the transformation of oxidosqualene to a predominant protostadienol along with a minor triterpenoid and that $C f a O S C 2$ was associated with protostane-type triterpenoid biosynthesis using a heterologous expression system in yeast cells. However, the next catalytic step of the cycling triterpenoid remains unclear. Based on our DNA-sequence database, future studies need to elucidate the function of candidate genes in the gene cluster associated with CfaOSC2. In addtion, it will be necessary to identify $16 \beta$-acetyloxy-29-norprotosta1,17(20)Z,24-trien-3-one-21-oic acid or any triterpene compound in C. farinosa cells. Results 
of this study provide a greater understanding about biosynthetic mechanisms underlying steroidal triterpenoid biosynthesis in C. farinosa and other fungi.

Supplementary Materials: The following are available online at https:/ / www.mdpi.com/article/10 .3390 /genes12060848/s1, Figure S1: Putative fragmentation mechanism of protostadienol leading to the characteristic fragment ions observed in the GC-MS spectrum, Figure S2: HPLC analysis of helvolic acid. Two chromatograms of HPLC analysis, (a) an authentic helvolic acid and (b) the extract from C. farinosa mycelium.

Author Contributions: G.-H.A. carried out yeast experiments and GC-MS analysis. J.-G.H., H.-S.P. and G.-H.S. participated in DNA-sequencing analysis. O.-T.K. planned the work and wrote the manuscript. All authors have read and approved the final version of the manuscript.

Funding: This work was supported by a grant (PJ01194901) from the Next-Generation BioGreen 21 Program funded by Rural Development Administration, Republic of Korea.

Informed Consent Statement: Not applicable.

Data Availability Statement: The data presented in this study are available on request from the corresponding author.

Conflicts of Interest: The authors declare no conflict of interest.

\section{References}

1. Radwan, F.F.Y.; Hossain, A.; God, J.M.; Leaphart, N.; Elvington, M.; Nagarkatti, M.; Tomlinson, S.; Haque, A. Reduction of myeloid-derived suppressor cells and lymphoma growth by a natural triterpenoid. J. Cell. Biochem. 2015, 116, 102-114. [CrossRef] [PubMed]

2. Sun, K.X.; Xia, H.W. Pachymic acid inhibits growth and induces cell cycle arrest and apoptosis in gastric cancer SGC-7901 cells. Oncol. Lett. 2018, 16, 2517-2524. [CrossRef]

3. Zhao, S.; Gao, Q.; Rong, C.; Wang, S.; Zhao, Z.; Liu, Y.; Xu, J. Immunomodulatory effects of edible and medicinal mushrooms and their bioactive immunoregulatory products. J. Fungi 2020, 6, 269. [CrossRef] [PubMed]

4. Lv, J.M.; Hu, D.; Gao, H.; Kushiro, T.; Awakawa, T.; Chen, G.D.; Wang, C.X.; Abe, I.; Yao, X.S. Biosynthesis of helvolic acid and identification of an unusual C-4-demethylation process distinct from sterol biosynthesis. Nat. Commun. 2017, 8, 1644. [CrossRef]

5. Cao, Z.; Li, S.; Lv, J.; Gao, H.; Chen, G.; Awakawa, T.; Abe, I.; Yao, X.; Hu, D. Biosynthesis of clinically used antibiotic fusidic acid and identification of two short-chain dehydrogenase/reductases with converse stereoselectivity. Acta Pharm. Sin. B 2019, 9 , 433-442. [CrossRef] [PubMed]

6. Lodro, S.; Xiong, Q.; Wilson, W.K.; Ivanova, Y.; Smith, M.L.; May, G.S.; Matsuda, S.P. Protostadienol biosynthesis and metabolism in the pathogenic fungus Aspergillus fumigatus. Org. Lett. 2009, 11, 1241-1244. [CrossRef] [PubMed]

7. Mitsuguchi, H.; Seshime, Y.; Fujii, I.; Shibuya, M.; Ebizuka, Y.; Kushiro, T. Biosynthesis of steroidal antibiotic fusidanes: Functional analysis of oxidosqualene cyclase and subsequent tailoring enzymes from Aspergillus fumigatus. J. Am. Chem. Soc. 2009, 131, 6402-6411. [CrossRef]

8. Fei, Y.; Zhang, D.H.; Xu, J.W. Increased production of ganoderic acids by overexpression of homologous farnesyl diphosphate synthase and kinetic modeling of ganoderic acid production in Ganoderma lucidum. Microb. Cell Factories 2019, 18, 115. [CrossRef]

9. Abe, I.; Prestwich, G.D. Molecular cloning, characterization, and functional expression of rat oxidosqualene cyclase cDNA. Proc. Natl. Acad. Sci. USA 1995, 92, 9274-9278. [CrossRef]

10. Joffrion, T.M.; Collins, M.S.; Sesterhenn, T.; Cushion, M.T. Functional characterization and localization of Pneumocystis carinii lanosterol synthase. Eukaryot. Cell 2010, 9, 107-115. [CrossRef] [PubMed]

11. Shang, C.H.; Shi, L.; Ren, A.; Qin, L.; Zhao, M.W. Molecular cloning, characterization, and differential expression of a lanosterol synthase gene from Ganoderma lucidum. Biosci. Biotechnol. Biochem. 2010, 74, 974-978. [CrossRef] [PubMed]

12. Shi, Z.; Buntel, C.J.; Griffin, J.H. Isolation and characterization of the gene encoding 2,3-oxidosqualene-lanosterol cyclase from Saccharomyces cerevisiae. Proc. Natl. Acad. Sci. USA 1994, 91, 7370-7374. [CrossRef]

13. Suzuki, M.; Xiang, T.; Ohyama, K.; Seki, H.; Saito, K.; Muranaka, T.; Hayashi, H.; Katsube, Y.; Kushiro, T.; Shibuya, M.; et al. Lanosterol synthase in dicotyledonous plants. Plant Cell Physiol. 2006, 47, 565-571. [CrossRef] [PubMed]

14. Du, C.; Wu, J.; Cuthbertson, A.G.; Bashir, M.H.; Sun, T.; Ali, S. Morphological, molecular and virulence characterisation of six Cordyceps spp. isolates infecting the diamondback moth, Pluttela xylostella. Biocontrol Sci. Technol. 2021, 31, 373-386. [CrossRef]

15. Zhao, M.; Godecke, T.; Gunn, J.; Duan, J.A.; Che, C.T. Protostane and fusidane triterpenes: A Mini-Review. Molecules 2013, 18, 4054-4080. [CrossRef]

16. Hikino, H.; Asada, Y.; Arihara, S. Fusidic acid, a steroidal antibiotic from Isaria kogane. Chem. Pharm. Bull. 1972, 20, 1067-1069. [CrossRef]

17. Zerbino, D.R.; Birney, E. Velvet: Algorithms for de novo short read assembly using de Bruijn graphs. Genome Res. 2008, 18, 821-829. [CrossRef] 
18. Altschul, S.F.; Gish, W.; Miller, W.; Myers, E.W.; Lipman, D.J. Basic local alignment search tool. J. Mol. Biol. 1990, 215, 403-410. [CrossRef]

19. Sievers, F.; Wilm, A.; Dineen, D.G.; Gibson, T.J.; Karplus, K.; Li, W.; Lopez, R.; McWilliam, H.; Remmert, M.; Söding, J.; et al. Fast, scalable generation of high-quality protein multiple sequence alignments using Clustal Omega. Mol. Syst. Biol. $2011,7,539$. [CrossRef]

20. Tamura, K.; Stecher, G.; Peterson, D.; Filipski, A.; Kumar, S. MEGA6: Molecular evolutionary genetics analysis version 6.0. Mol. Biol. Evol. 2013, 30, 2725-2729. [CrossRef] [PubMed]

21. Kushiro, T.; Shibuya, M.; Ebizuka, Y. $\beta$-amyrin synthase-Cloning of oxidosqualene cyclase that catalyzes the formation of the most popular triterpene among higher plants. Eur. J. Biochem. 1998, 256, 238-244. [CrossRef] [PubMed]

22. Wang, Z.; Yeats, T.; Han, H.; Jetter, R. Cloning and characterization of oxidosqualene cyclases from Kalanchoe daigremontiana: Enzymes catalyzing up to 10 rearrangement steps yielding friedelin and other triterpenoids. J. Biol. Chem. 2010, 285, 29703-29712. [CrossRef] [PubMed]

23. Moreno-Hagelsieb, G.; Latimer, K. Choosing BLAST options for better detection of orthologs as reciprocal best hits. Bioinformatics 2008, 24, 319-324. [CrossRef]

24. Kepler, R.M.; Luangsa-Ard, J.J.; Hywel-Jones, N.L.; Quandt, C.A.; Sung, G.H.; Rehner, S.A.; Aime, M.C.; Henkel, T.W.; Sanjuan, T.; Zare, R.; et al. A phylogenetically-based nomenclature for Cordycipitaceae (Hypocreales). IMA Fungus 2017, 8, 335-353. [CrossRef]

25. Poralla, K.; Hewelt, A.; Prestwich, G.D.; Abe, I.; Reipen, I.; Sprenger, G. A specific amino acid repeat in squalene and oxidosqualene cyclases. Trend Biochem. Sci. 1994, 19, 157-158. [CrossRef]

26. Kimura, M.; Kushiro, T.; Shibuya, M.; Ebizuka, Y.; Abe, I. Protostadienol synthase from Aspergillus fumigatus: Functional conversion into lanosterol synthase. Biochem. Biophys. Res. Commun. 2010, 391, 899-902. [CrossRef] 